\title{
Focal Plane Array Spectrometer FPAS: preliminary development results and recommendations
}

M. Madi, G. Osowiecki, E. Alberti, H. P. Herzig

M. Madi, G. Osowiecki, E. Alberti, H. P. Herzig, "Focal Plane Array Spectrometer FPAS: preliminary development results and recommendations," Proc. SPIE 11852, International Conference on Space Optics - ICSO 2020, 118526V (30 June 2021); doi: 10.1117/12.2604387

SPIE Event: International Conference on Space Optics - ICSO 2021, 2021, Online Only 


\section{International Conference on Space Optics-ICSO 2020}

Virtual Conference

30 March-2 April 2021

Edited by Bruno Cugny, Zoran Sodnik, and Nikos Karafolas
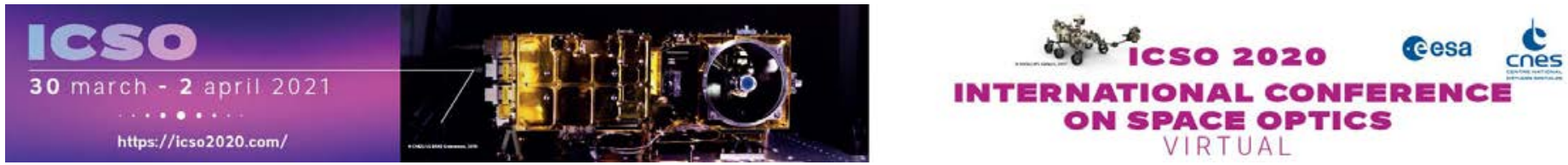

\section{Focal Plane Array Spectrometer FPAS: preliminary development results and recommendations}

\section{eesa isso procedings denes}




\title{
Focal Plane Array Spectrometer FPAS: preliminary development results and recommendations
}

\author{
Madi M.*a,b, Osowiecki G. ${ }^{\text {b }}$, Alberti E. ${ }^{\mathrm{a}}$, and Herzig H. P. ${ }^{\mathrm{b}}$ \\ aMicos Engineering GmbH, Überlandstrasse 129, CH-8600 Dübendorf, Switzerland \\ ${ }^{\text {b} E c o l e ~ P o l y t e c h n i q u e ~ F e ́ d e ́ r a l e ~ d e ~ L a u s a n n e ~(E P F L), ~ O p t i c s ~ a n d ~ P h o t o n i c s ~ T e c h n o l o g y ~}$ \\ Laboratory, Neuchâtel CH-2000, Switzerland
}

\begin{abstract}
The focal plane array spectrometer (FPAS) is a miniaturization concept for imaging spectrometers compared to classical dispersive and FTS instruments. FPAS is an imaging implementation of interferogram integrated FTS, targeting space-borne and commercial applications. It is based on a bi-dimensional array of waveguide spectrometers which can be assembled in small size, and form a compact package of single spectrometers. When this system is positioned in the focal plane of an objective (in a similar way as a CMOS detector array or a CCD in a camera), it will allow imaging spectrometry of the observed surface (objects). The instrument is therefore reduced to the imaging optics and the FPAS in the focal plane, which takes over the role of spectrometer and detector array. This is a breakthrough concept enabling imaging spectroscopy in a reduced volume with low power consumption. This article describes the preliminary development results of a FPAS based on arrays of single-mode waveguides. The developed linear array forms an acquisition line to image with lateral scanning. The light is injected with micro-lenses into several optical polymer waveguides. In order to circumvent the sub-sampling limitation due to nano-sampler spacing and to expand the spectral bandwidth, the basic principle of a static Lippmann spectrometer is combined with a dynamic Fourier-transform spectrometer, by adopting a piezo actuated movable mirror located at the waveguide end-facet. This waveguide spectrometer is designed for a nominal bandwidth of $25 \mathrm{~nm}$ at central wavelength of $762.5 \mathrm{~nm}$ and a spectral resolution of about 0.06 $\mathrm{nm}$. The throughput and SNR in this preliminary linear array prototype are analysed and design limitations are discussed. The authors then introduce a new method for the realization of FPAS aiming at enhancing throughput and bandwidth of the integrated device. This new concept uses a bundle of single-mode waveguides, instead of only one, at the focal distance of the optical element. The novel FPAS concept is elaborated and its characteristics are described.
\end{abstract}

Keywords: multispectral and hyperspectral imaging, fourier transform spectrometers, focal plane arrays, photonic crystal waveguides

\section{INTRODUCTION}

Focal plane array spectrometer (FPAS), introduced in 2010 by Guldimann and Kraft, ${ }^{1}$ is a concept for a miniaturized spectrometer compared to classical dispersive and FTS instruments. FPAS is an imaging implementation of interferogram integrated FTS, targeting space-borne and commercial applications. It is based on a bi-dimensional array of waveguide spectrometers which can be assembled in small size, and form a compact package of single spectrometers. When this system is positioned in the focal plane of an objective - in a similar way as a CMOS detector array or a CCD in a camera - it will allow imaging spectrometry of the observed surface (objects). ${ }^{1}$ The instrument is therefore reduced to the imaging optics and the FPAS in the focal plane, which takes over the role of spectrometer and detector array. ${ }^{1}$ This is a breakthrough concept enabling imaging spectroscopy in a reduced volume with low power consumption.

A single block (pixel) of FPAS includes a single-mode waveguide, nano-samplers in the evanescent field and a compact photodetector. The light injected in the single-mode optical waveguide with a reflective surface (mirror)

Corresponding authors:

Madi M., E-mail: mmadi@gmx.ch, Herzig H. P., E-mail: hanspeter.herzig@epfl.ch 
at its end generates an interference pattern, called interferogram, along the waveguide. ${ }^{1}$ This phenomenon generates a stationary wave (interferogram). The energy extraction required to sample the standing wave is obtained by sampling the evanescent field on one side of the waveguide using nano-samplers - also called evanescent field samplers or antennas - located in the evanescent field. ${ }^{2-6}$ These nano-samplers scatter the light around an axis perpendicular to that of propagation of the waveguide. For each nano-sampler, the scattered light is detected by a pixel aligned with this axis. The intensity detected is thus proportional to the intensity of the interferogram at the exact location of the nano-sampler. A mathematical apparatus, based on Fourier transform, takes into account all the calibration data and, when applied to the linear image, gives the spectrum of the light.

In the original concept of FPAS as shown in Fig. 1, single-mode waveguides are placed in the focal plane of the imaging optics. The radiation is collected by the telescope, focused on the optical elements (e.g. microlenses) which couple the light into waveguides. The amount of light injectable into the system is intrinsically limited due to the small capacity of individual waveguides (see Sec. 3). Limited throughput at the level of individual waveguides limits the system's overall signal-to-noise ratio (SNR).

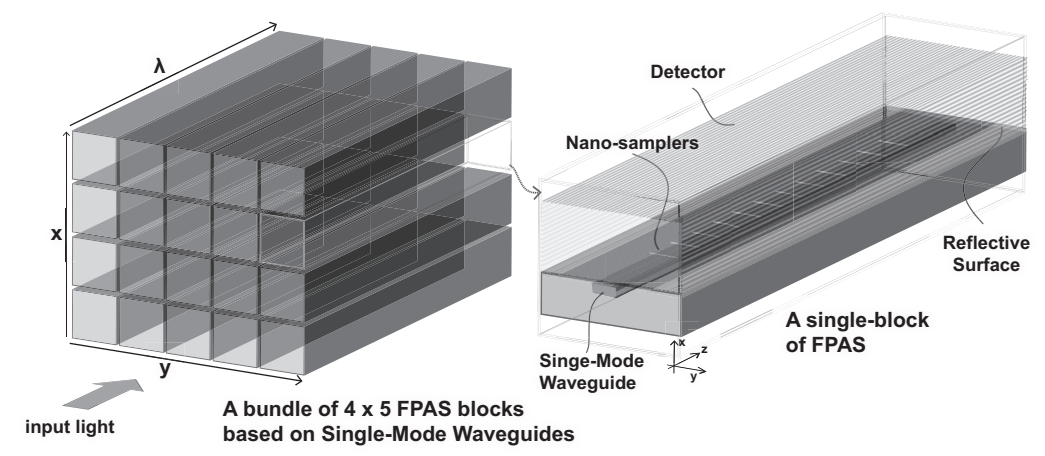

Figure 1. Layout of the FPAS on-chip as proposed in. ${ }^{1}$ Here a block of $4 \times 5$ sub-arrays are shown. Each pixel of the array contains a waveguide spectrometer including a single-mode waveguide, patterns of evanescent field samplers, and a compact photodetector. The radiation is collected from the scene (xy plane) and imaged on the focal plane of the telescope where the arrays of FPAS are located. The spectral information is extracted along the waveguides (marked as $\lambda$ in the block). Hence, the output is a 3D-image with two spatial axes reflecting the scene and one axis containing spectral information of the scene, i.e. each pixel in the output image contains a sampled spectral measurement of reflectance.

Ideally, miniaturization of a spectrometer devices should not limit its light gathering capability or otherwise compromise its performance. ${ }^{7}$ In many spectroscopy applications, specifically in the space domain, a large optical throughput and a high spectral resolution is needed. Thereby, the efforts are concentrated on the development of compact imaging spectrometers with high spectral resolution, large bandwidth and high throughput.

In Sec. 2, we analyse the performance of a linear array of single-mode waveguides which is the first realization of FPAS. In Sec. 3, the throughput and SNR in the developed linear array spectrometer is studied and methods to improve them are described in Sec. 4.1. Followed by that, in Sec. 4.2, an innovative concept for the development of a FPAS system with enhanced throughput and performance is introduced.

\section{FPAS DEVELOPMENT BASED ON ARRAYS OF SINGLE-MODE WAVEGUIDES}

In order to investigate the feasibility of FPAS concept, in the frame of a Technology Research Programme (TRP) funded by the European Space Agency (ESA), we fabricated a linear array of waveguide spectrometers forming an acquisition line to image with lateral scanning. ${ }^{8,9}$ In this standing wave integrated Fourier transform spectrometer, light is injected with microlenses into several optical polymer waveguides. In order to circumvent the sub-sampling limitation due to nano-sampler spacing and to expand the spectral bandwidth of the recollected spectrum, the basic principle of a static Lippmann spectrometer is combined with a dynamic Fourier-transform spectrometer, by adopting a piezo actuated movable mirror located at the waveguide end-facet, which introduces small optical path difference (OPD) changes. Overall, the use of a movable mirror in this FPAS prototype increases the size and complexity of the waveguide spectrometer system. This approach is spiritually against 
the miniaturization concept which is the core of FPAS. In the following subsections, the performance of this prototype is analysed.

\subsection{Monochromatic spectrum measurements: calibration and data analysis}

For the analysis of the linear-array spectrometer performance, we used a sample similar to that of figure 2 in Osowiecki et al. ${ }^{8}$ The sample contains single-mode waveguides in a line with a spacing of $250 \mu \mathrm{m}$ due to microlens diameter. The signal cross-talk between the neighbouring waveguides in the array has been experimentally proved to be very low, thereby negligible. The evanescent field samplers are gold nanorods with optimized dimensions for the band of interest $\left(750 \mathrm{~nm}-775 \mathrm{~nm}\right.$ ), i.e. $50 \mathrm{~nm}$ (width), $20 \mathrm{~nm}$ (thickness) and $8 \mu \mathrm{m}$ (length). ${ }^{10}$ The samplers are fabricated longer than the waveguide's width to make sure that they cover the entire width of the waveguide, for assuring an effective interaction between samplers and the evanescent field. The gold samplers are $10 \mu \mathrm{m}$ apart and 180 samples cover a distance of about $1.8 \mathrm{~mm}$ on the waveguide. This sampling distance in EpoClad/EpoCore ${ }^{11}$ waveguide with an effective refractive index $\left(\mathrm{n}_{\mathrm{eff}}\right)$ of 1.575 at the central wavelength $(762.5$ $\mathrm{nm})$ provides a spectral resolution of $\mathrm{R} \approx 7,500(\Delta \lambda \approx 0.06 \mathrm{~nm})$. Figure 2 shows a chip under test in the state when the light is coupled into the waveguide arrays. It is worthwhile mentioning that the band of interest, 750 $\mathrm{nm}-775 \mathrm{~nm}$, is a design requirement in the TRP activity. Nevertheless, in Sec. 2.2, the broadband tests are performed in a shifted band, $765 \mathrm{~nm}-785 \mathrm{~nm}$, given by the test equipment availability.

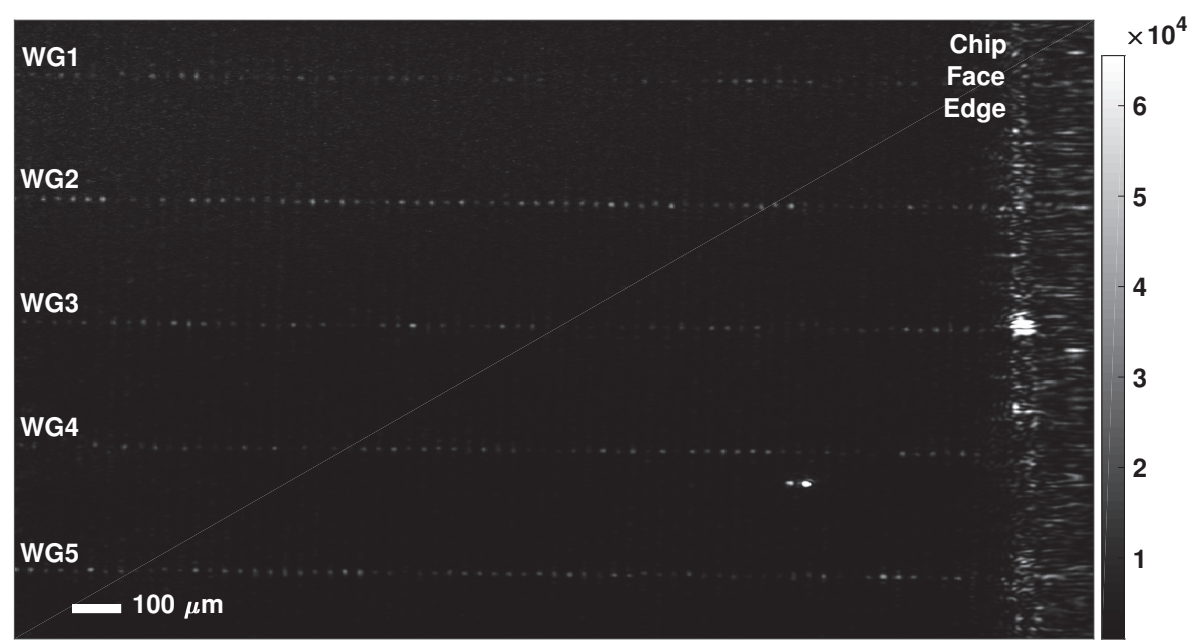

Figure 2. The chip under test contains 5 single-mode waveguides in a linear array with $250 \mu \mathrm{m}$ spacing. Evanescent field samplers are fabricated at an interval bigger than $10 \mathrm{~nm}$ in the shown chip. The light is injected into the waveguides from the right side through an array of microlenses (not shown here). The shining points along the horizontal lines are the scattered light from the evanescent field samplers located on the top of the waveguide core.

For testing the system performance, two monochromatic laser sources are used as the reference spectrum, including a Thorlabs LP660-SF60 laser diode driven in constant current mode at $120 \mathrm{~mA}$, and an external cavity tunable laser diode driven at $1.2 \mathrm{~A}$. A set of calibration attempts are undertaken prior to signal processing. For this purpose, two sets of images are captured at the wavelengths of $660 \mathrm{~nm}$ and $790 \mathrm{~nm}$ with the reflecting mirror placed at one end of the chip (left side of the chip in Fig. 2). These images are used for calibrating the evanescent field samplers position by phase shift analysis. The reflecting mirror is driven with a closed loop piezo-actuator with the steps of $20 \mathrm{~nm}$. Thereby, at least 500 images are recorded to cover $10 \mu \mathrm{m}$ pitch of samplers. Extra measurements without mirror movements at different integration times (500 ms, 1,000 ms, $1,500 \mathrm{~ms}$, and 5,000 ms) and two wavelengths of $660 \mathrm{~nm}$ and $790 \mathrm{~nm}$ have been performed for calibrating samplers scattering efficiency. In addition, the measurements without reflecting mirror are done to estimate the offset in the measured interferogram due to propagation loss and backscattering of evanescent field samplers. Figure 3 shows a part of sampled interferogram - $0.62 \mathrm{~mm}$ long — extracted from the five waveguides of Fig. 2. 


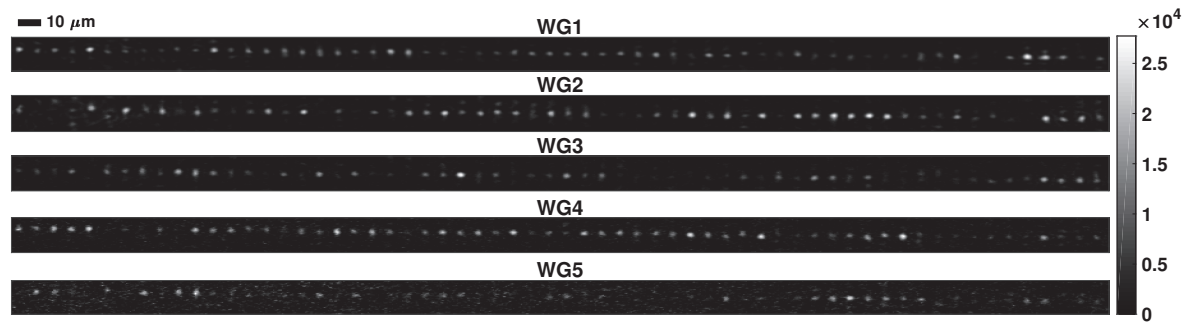

Figure 3. The sampled interferogram at $790 \mathrm{~nm}$ as extracted from the individual waveguides (WGs) in the linear array of Fig. 2.

The evanescent field samplers are fabricated in e-beam lithography. ${ }^{12}$ The SEM inspection has revealed that the nanorods look more similar to an enlarged potato than a uniform rod because the gold crystals are almost $10 \mathrm{~nm}$ in diameter and each nanorod consists of several gold crystals. ${ }^{12,13}$ Likewise, as depicted in Fig. 4, the scattering efficiencies are not alike as the geometrical factors of individual samplers are different. For capturing the interferogram data of Fig. 4, the monochromatic laser at $790 \mathrm{~nm}$ is coupled into waveguides and the scattered light out of each individual evanescent field sampler is recorded in a batch of 500 images each corresponding to a mirror step. The scattering efficiencies of samplers are calibrated and compensating factors are generated prior to spectrum reconstruction.

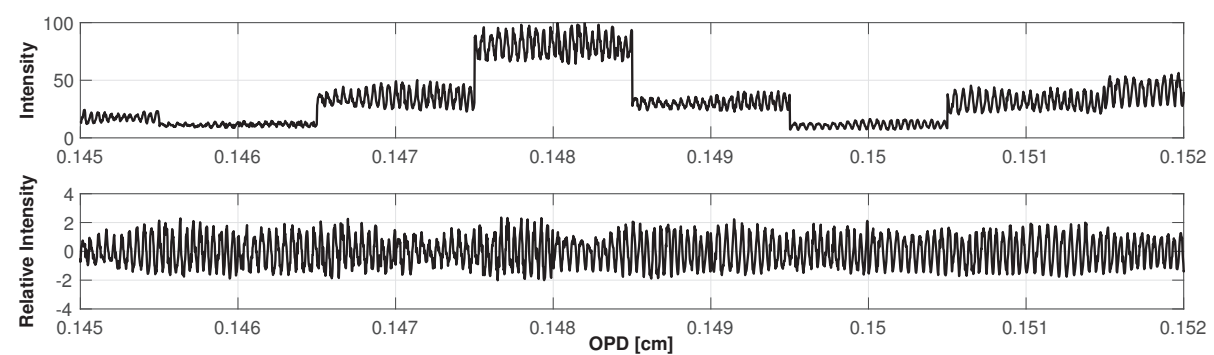

Figure 4. The top diagram shows the sampled interferogram from 8 consecutive evanescent field samplers. The lower diagram shows the same interferogram after correcting the samplers' scattering efficiencies and normalizing the amplitudes.

The reconstructed spectrum out of $1.24 \mathrm{~mm}$ part of the interferogram is depicted in Fig. 5. The spectral side glitches arising from retro-reflections and energy dissipation in waveguide in combination with the mirror steps. The sampled data from all five waveguides have been integrated in order to increase the SNR.

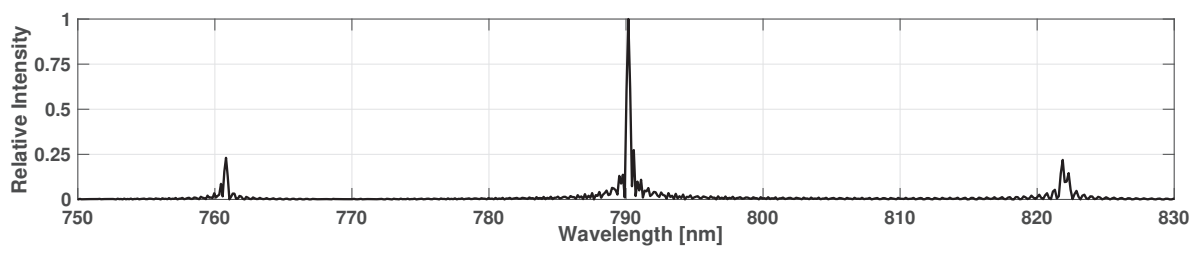

Figure 5. The reconstructed spectrum out of the sampled interferogram as partially shown in Fig. 4.

\subsection{Polychromatic spectrum measurements: test setup preparation, modelling and performance verification}

In most science applications, the input to the spectrometer is a polychromatic light which may contain absorption signatures of particles. A super-continuum laser source from NKT Photonics was used to provide the broadband spectrum for the tests. The spectral management setup is shown in Fig. 6; it contains the light source, filters and couplers. Interference filters are used to adjust the spectrum to the desired band. With a double interference filter set, the necessary test spectrum could be obtained. We used two Semrock bandpass filters and one OptiGrate 
notch filter. By rotating these high quality filters, it is possible to move their transmission bands to the lower wavelength side and adjust bandwidth and position of the notch filter peak which represents the absorption signature. The super-continuum laser source is stabilized in power with internal feedback and power lock to provide a stable spectral reference.

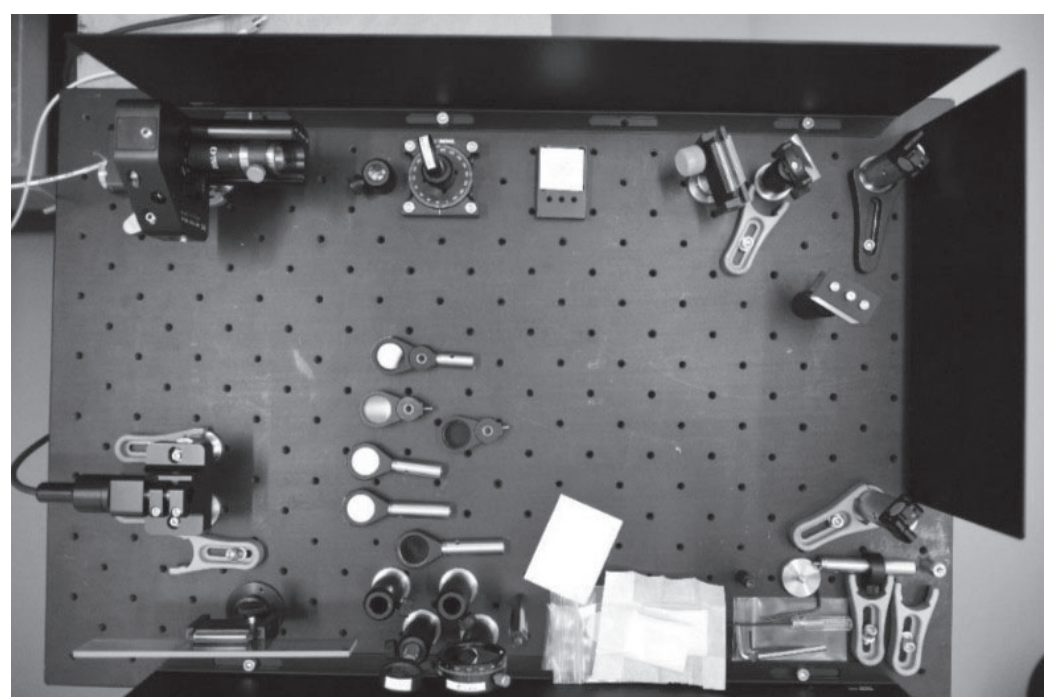

Figure 6. Spectral management system containing light entrance from the super-continuum laser source (lower left corner), filters on rotational supports (upper row) and fiber coupler (upper left corner).

The constructed reference broadband spectrum is measured and shown in Fig. 7. The location of the absorption signature can be adjusted via the notch filter.

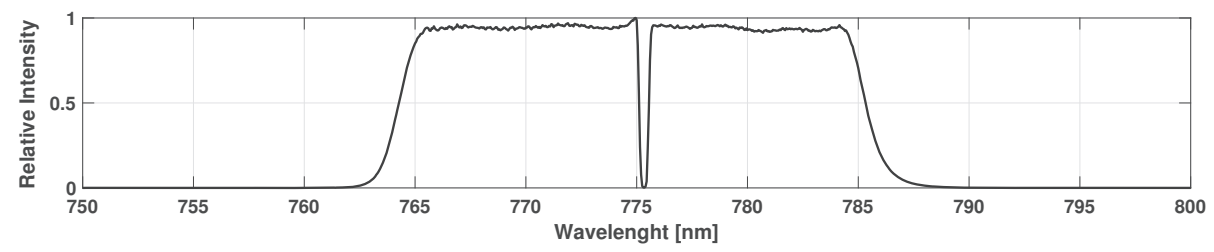

Figure 7. Reference broadband spectrum with absorption line as the output of spectral management system (Fig. 6): $20 \mathrm{~nm}$ bandpass centred at $775 \mathrm{~nm}$ with notch filter set at $\sim 775 \mathrm{~nm}$.

The polychromatic interferogram corresponding to the spectrum of Fig. 7 has been modelled and studied before testing the spectrometer performance, in order to prepare the calibration algorithms and comprehend the expected results. A broadband interferogram has its maximum at the zero optical path difference (ZPD), and the interferogram adjacent to ZPD contains the main broadband information (see Fig. 8).

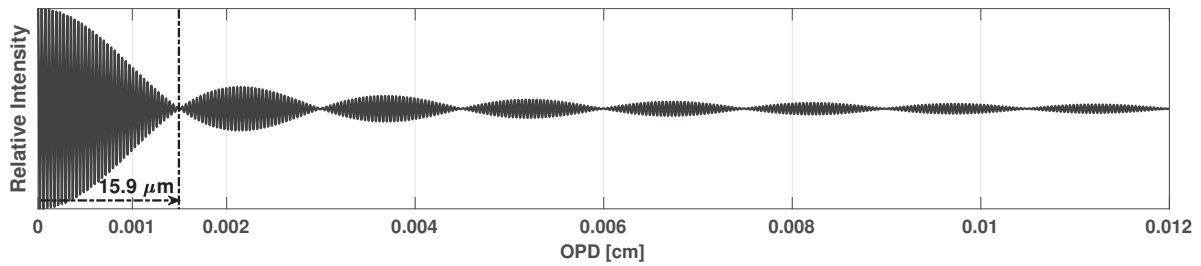

Figure 8. Simulation of interferogram from a $20 \mathrm{~nm}$ broadband signal $(765 \mathrm{~nm}-785 \mathrm{~nm})$ without absorption signature in EpoClad/EpoCore medium.

The modelling shows that when a signal with the broadband spectrum similar to that of Fig. 7 is coupled 
into the waveguide spectrometer arrays, the polychromatic interferogram faces attenuations of various sources. Geometrical limitations in perfectly aligning the mirror parallel to the waveguide end face and perpendicular to the waveguides on the chip led to a relatively large minimum air gap of about $5 \mu \mathrm{m}$. That leads to the loss of the interferogram part that lies in the mirror-waveguide air gap. In addition, since the distance between the mirror and the chip edge varies per mirror step, the loss in the air gap varies as well. This is compensated by introducing adaptive correction factors.

Figure 9 is a numerical modelling summarizing the three sources of attenuation and their impacts on the interferogram. The propagation loss in the waveguide represents itself as an offset whereas the air gap attenuates the signal at various rates depending on the gap dimension. Prior to spectrum reconstruction, the sampled interferogram data is calibrated and the attenuations are compensated.

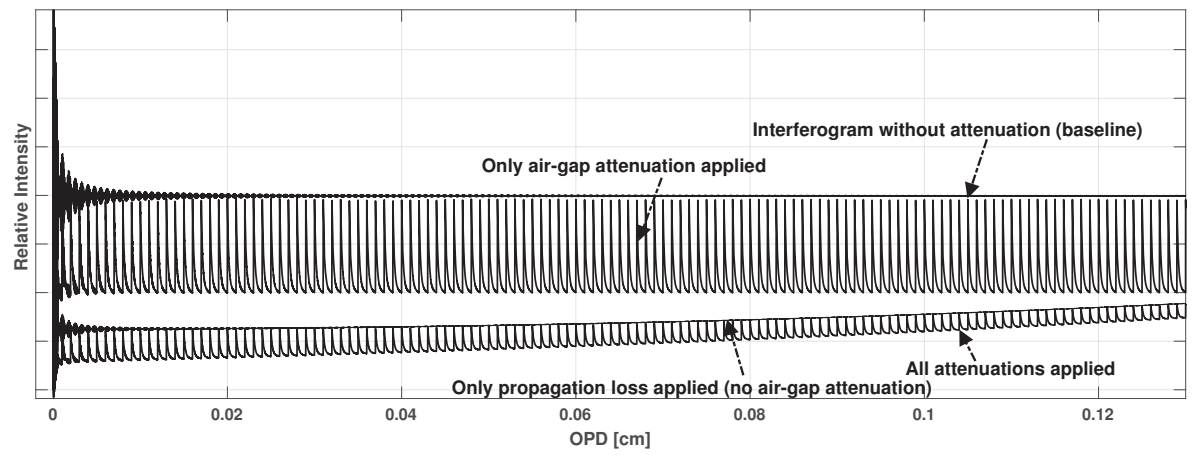

Figure 9. Numerical modeling showing the polychromatic interferogram affected by attenuations of different types.

In waveguide spectrometers based on the Lippmann interference concept where the reflective surface is located at one end of the waveguide, ZPD is exactly at the position of the reflective surface. For this reason, the ZPD data is practically unretrievable in the state-of-the-art technology as the evanescent field samplers cannot be fabricated exactly on the reflective surface. In the waveguide spectrometer prototype under test, the loss of ZPD is not only because of the evanescent field samplers positioning but also because of the air gap between the waveguide edge face and the mirror. The loss of ZPD and its neighbourhood up to some microns is not crucial for spectrum reconstruction of monochromatic lines, e.g. laser sources with narrow-bandline. However, it is an issue for Lorentzian lines or polychromatic sources, knowing that the main lobe of the sinc-function, as the envelope of the broadband data, has a very short coherent length. The coherence length of light in EpoCore/EpoClad medium for $20 \mathrm{~nm}$ spectral width of the light source with central wavelength $(\lambda)$ at $775 \mathrm{~nm}$ is given in Eq. (1):

$$
L=\sqrt{\frac{2 \ln (2)}{\pi \mathrm{n}_{\mathrm{eff}}}} \times \frac{\lambda^{2}}{\Delta \lambda}
$$

The main lobe of the interferogram is about $15.9 \mu \mathrm{m}$ long according to Eq. (1). This is consistent with the simulation results which was shown in Fig. 8. If the source has a Gaussian spectrum with FWHM spectral width $(\Delta \lambda)$, a path offset of $\pm L$ will reduce the fringe visibility to $50 \%{ }^{14}$ The broader the spectrum, the shorter the coherent length of the corresponding interferogram.

The truncation of the interferogram near the ZPD and its effect on the reconstructed spectrum is simulated in the following. The reference spectrum of Fig. 7 is first converted into interferogram, then the main lobe data near the ZPD (a length of $15.9 \mu \mathrm{m}$ ) is truncated and the resulted interferogram is transferred back to the spectral domain. The dotted line in Fig. 10 is the resulting spectrum which contains the signature of the absorption line but with corrupted phase information.

Thereby, for the broadband spectrum of Fig. 7 with an absorption line, the loss of data near ZPD is equal to losing the broadband envelope. Nevertheless, the simulation of Fig. 10 showed that the signature of the absorption line is in the tail of the interferogram and it is retrievable in the interferogram domain with corrupted phase information. For examining this phenomenon, the super-continuum laser source of Fig. 6 was coupled 


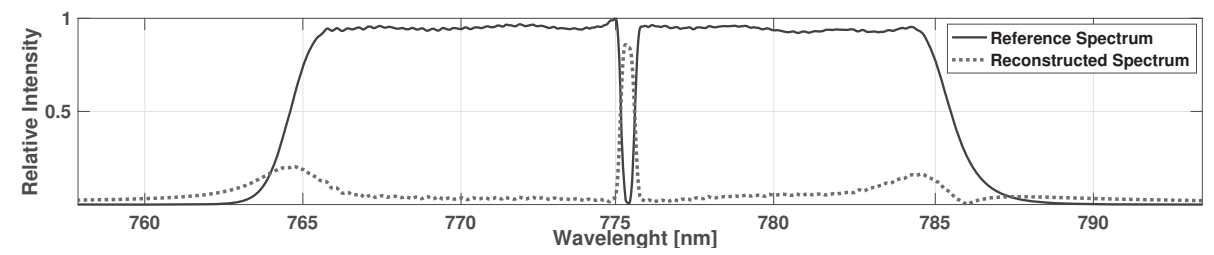

Figure 10. The reference broadband spectrum with an absorption line is shown in solid line and the reconstructed spectrum from a corresponding interferogram after ZPD data truncation is shown in dotted line.

into the waveguide spectrometer array, and the tail of the interferogram was sampled. Figure 11 shows the reconstructed spectrum which despite being extremely noisy, contains the signature of the absorption line.

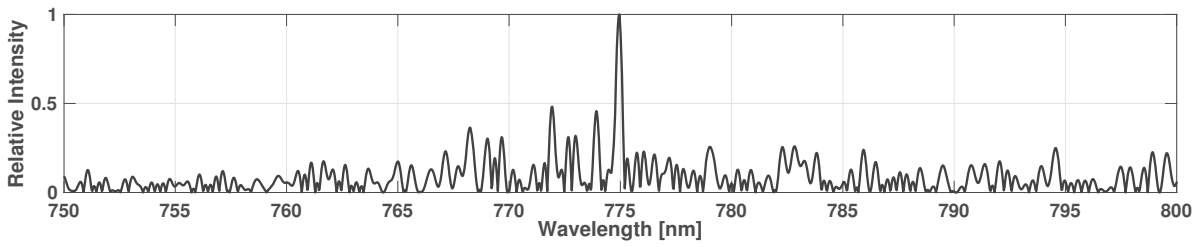

Figure 11. Reconstructed spectrum from $0.6 \mathrm{~mm}$ long polychromatic interferogram tail corresponding to the supercontinuum laser source of Fig. 7 (without ZPD information).

\section{THROUGHPUT IN WAVEGUIDE SPECTROMETERS}

The maximum amount of light that can be coupled into the waveguide as an optical device is determined by $B_{e} \times S_{\min }$, where $B_{e}$ is the radiance of the light emitter (source) and $S_{\min }$ is the throughput of the source or the optical device, whichever is smaller. ${ }^{15}$ In science applications, e.g. remote sensing, the received radiance $\left(\mathrm{W} / \mathrm{sr}^{\mathrm{m}} \mathrm{m}^{2}\right)$ at the aperture of the telescope aboard the satellite is very small. Hence, the aim is to couple as much radiance as possible into each individual waveguide in the focal plane. This in its turn has a strong impact on the signal to noise ratio (SNR) in a waveguide spectrometer.

For a waveguide, throughput is proportional to the number of orthogonal modes supported by the structure. ${ }^{15}$ It may be emphasized that once the cut-off frequency for single-mode operations in certain waveguide materials is given, the throughput of the waveguide is constant through different varying parameters. Therefore, the application of single-mode waveguides in the original FPAS design concept ${ }^{1}$ is accompanied by setting a fixed and limited system throughput. Consequently, the developed prototype suffers from the limited throughput capacity of the whole system because the collected photons from the aperture cannot be efficiently coupled into the spectrometer system. Limited throughput at the level of individual waveguides limits the system's overall signal-to-noise ratio (SNR).

The throughput in waveguide spectrometers depends also on the coupling efficiency of the input optics. Nevertheless, it is dominated by the parameters of the waveguide. Three factors restrict the throughput of the system, 1) intrinsic throughput limitation of single-mode waveguides, 2) fill factor of microlenses used in such design, and 3) the energy loss while coupling from microlenses into single-mode waveguides. In the following, we explain how these factors limit the spectrometer throughput and we calculate the estimated SNR for the FPAS prototype.

\subsection{Coupling efficiency in FPAS}

In the state-of-the-art FPAS configuration, as shown in Fig. 12, microlenses are placed in the focal plane of the telescope (here only two cells of FPAS are shown). The microlenses are arranged in a line and have a circular aperture with radius $r$. The fill factor of microlenses is $\pi r^{2} /\left(4 r^{2}\right)=\pi / 4(\sim 78.5 \%)$ in the best case scenario. Furthermore, Guldimann and $\mathrm{Kraft}^{1}$ show that the energy coupling between a microlens and a singlemode waveguide is always lossy and the coupling efficiency, CEF\#1 in Fig. 12, is less than 60\% (down to 10\%). Thereby, the overall coupling efficiency in the original FPAS design is less than 50\%; this is the best case scenario. 


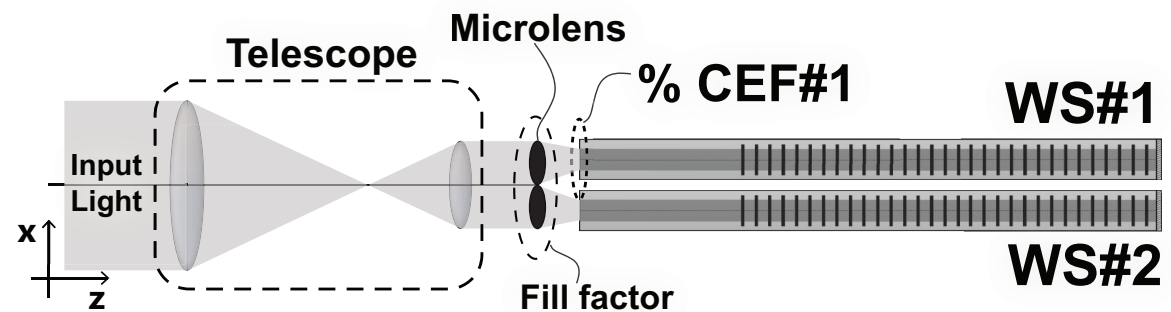

Figure 12. In the state-of-the-art configuration, coupling optics (e.g. microlenses) are placed in the focal plane of the telescope to couple the light into single-mode waveguides. The fill factor of the system is $\sim 78.5 \%$ in the best case. Here only two pixels of the FPAS array are shown.

\subsection{SNR in FPAS}

The SNR for the detection of a specific spectral line is derived from the overall model of the FPAS system and depends on a number of parameters including the mean value of the signal over the entire spectrum and the dark noise on the detector pixel. In a FPAS, the mean value of the signal is evaluated from the incoming light in the waveguide, the efficiency of the evanescent field samplers, the collecting efficiency of the image transfer optics, and the quantum efficiency of the detector array. The value of dark noise is taken from the data sheet of the detector.

Numerical modeling shows that the SNR for the FPAS system is $\sim 500$ for a narrowband spectrum (monochromatic) and it is $\sim 50$ for a broadband spectrum (polychromatic). To give an idea about the market demand on SNR, the CarbonSat mission which was originally proposed to improve the global greenhouse gas monitoring capabilities, has a requested SNR of $>500$ in the NIR region.

The SNR increases with the square root of the total integration time. ${ }^{16}$ Thus, averaging the interferogram acquired over multiple acquisitions or multiple waveguides for the same target scene would in fact allow to increase the SNR. An alternative approach is to increase the incoming light in the waveguide; in other words, to improve the system throughput which is discussed in the following section.

\section{THROUGHPUT ENHANCEMENT IN WAVEGUIDE SPECTROMETER SYSTEMS}

\subsection{Efforts review}

One method to increase the SNR in FPAS is to improve the throughput of each individual waveguide spectrometer. Madi et al. ${ }^{10,12}$ have shown that the limited throughput in single-mode waveguides can be enhanced by optimization of the unguided light in the unconfined direction of the planar waveguide. In the proposed Lippmann planar waveguide spectrometer (LiPWS), the interferogram is formed within a planar waveguide instead of a single-mode channel waveguide. The mode expansion in planar waveguides provides a larger acceptance area, yielding a higher throughput in individual waveguide spectrometers. In addition, a customized pattern of nano-samplers fabricated on the surface of the planar waveguide has allowed to increase the measurement points of the interferogram yielding a wider spectral bandwidth in a fully static way. ${ }^{10} \mathrm{~A}$ waveguide spectrometer prototype made in silicon oxynitride/silicon dioxide technology has shown a nominal bandwidth of $256 \mathrm{~nm}$ thanks to a custom pattern of nanodisks providing $0.25 \mu \mathrm{m}$ sampling interval at central wavelength of $633 \mathrm{~nm}$. This is an innovative technique towards development of highly compact individual waveguide spectrometers with high bandwidth and spectral resolution. Though LiPWS concept has significant advantages, the overall throughput of a bundle of LiPWS in an array is limited.

In this sense, compact spectrometers in planar waveguides based on waveguide gratings have been developed. ${ }^{7,17,18}$ In order to increase the device throughput the grating concept is replaced by the interferometerbased spectrometer. ${ }^{19,20}$ Florjańczyk et al. ${ }^{21,22}$ have shown that the optical throughput is increased by a factor 
of 200 compared to single input spectrometer devices by using multiple input waveguide apertures which feed into an array of Mach-Zehnder interferometers. A multi-aperture planar waveguide spectrometer based on 50 arrayed Mach-Zehnder interferometers has provided spectral resolution of $0.1 \mathrm{~nm}$ over $2.5 \mathrm{~nm}$ bandwidth at $1.36 \mu \mathrm{m} .^{23}$ The optical input is formed by many independent planar waveguides, stacked in layers, providing an increase of throughput compared to single-waveguide input configurations. ${ }^{19}$ This device is, however, band-limited due to the fact that the maximum optical path difference which determines the spectral resolution is dictated by the stepwise imbalance introduced by the Mach-Zehnder interferometer integrated into the device. In these configurations, the optical path difference is formed on the detector array similarly to the classical Michelson interferometers. The only difference is the use of multi-aperture waveguides combined with Mach-Zehnder interferometers for creating optical path differences instead of moving parts. Notwithstanding the throughput advantage of the developed prototype, it is not appropriate to be used for imaging applications in FPAS configuration due to the complexity of the system and its dimension.

In Sec. 4.2, we introduce an innovative method for the development of FPAS which significantly improves the throughput of the waveguide spectrometer system and its overall performance.

\subsection{Innovative method}

In the new solution proposed by the authors, an array of multi-mode waveguides (e.g. multi-mode optical fiber) is placed in the focal plane of the telescope. Multi-mode waveguides have intrinsically higher throughput and acceptance angle compared to single-mode waveguides. Each multi-mode fiber accepts almost all the light which is coupled through the front-end optics (fill factor of MM-WG is $100 \%$ in Fig. 13) and couples the collected photons (with a certain coupling efficiency CEF\#2 in Fig. 13) into a bundle of single-mode fibers through a cluster of 3D photonic lanterns. Single-mode waveguides in their turns feed the waveguide spectrometers.

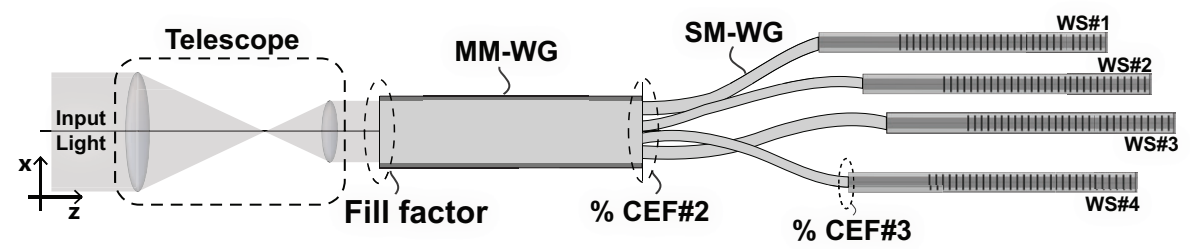

Figure 13. In the innovative configuration, a multi-mode waveguide (e.g. multi-mode fiber) is directly placed in the focal plane of the telescope. The fill factor of the system can be optimized to 100\%. This configuration can act as a self-standing waveguide spectrometer with the highest fill factor or as a single pixel of the innovative FPAS configuration.

According to the literature, the second law of thermodynamics does not allow lossless coupling of light from an arbitrarily excited multi-mode system into a single-mode one. ${ }^{24-27}$ But if the multi-mode system has the same number of degrees of freedom-same number of single-mode waveguides than number of modes in the multi-mode waveguide-lossless mode control and conversion becomes possible by keeping the entropy of the system.

The light in a multi-mode waveguide is converted into a system of multiple single-mode waveguides via an adiabatic transition. Conversions from multi-mode to single-mode and vice versa can have throughputs in excess of $90 \%$ (CEF\#2 in Fig. 13). ${ }^{24-27}$ This innovative solution is illustrated in Fig. 13, where MM, SM, WG and WS respectively stand for multi-mode, single-mode, waveguide and waveguide spectrometer.

Concerning the coupling of energy from the single-mode fiber to waveguide spectrometer, the coupling quality is very well correlated to the match between the fiber mode diameter and the waveguide geometric-mean diameter. ${ }^{28-31}$ show that a wide range of coupling techniques are currently being explored with an efficiency as high as 95\% (CEF\#3 in Fig. 13). Nevertheless, to reach a lossless coupling from a single-mode waveguide to a waveguide spectrometer, the single-mode waveguide itself can be used as the waveguide spectrometer (e.g. a D-shaped side polished optical fiber provides access to the evanescent field of the wave propagating in the optical fiber $^{32}$ ). In the latter case, the CEF \#3 is $100 \%$. Thus, the overall coupling efficiency in the configuration of Fig. 13 based on the multi-mode to single-mode concept can reach $80 \%$ to $90 \%$. 


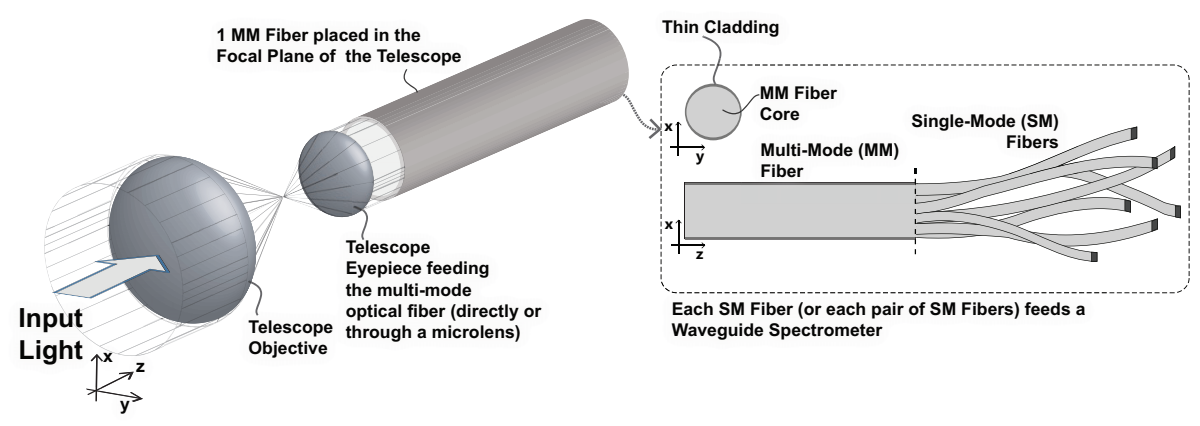

Figure 14. 3D visualization of a multi-mode waveguide spectrometer based on multi-mode round-core fiber with the highest fill factor acting as a self-standing waveguide spectrometer.

The multi-mode waveguide spectrometer can act as a self-standing spectrometer as shown in Fig. 14 or be integrated in an array for imaging applications as shown in Fig. 15. In case of a self-standing multi-mode waveguide spectrometer, a multi-mode round-core optical fiber can achieve the highest fill factor as it is placed in the focal distance of the telescope. Since a multi-mode fiber has a very large acceptance angle, the light from a microlens can be easily coupled into it. For imaging applications, an array of multi-mode square or rectangular-core optical fibers with very thin cladding layers can be arranged as shown in Fig. 15. Multi-mode optical fibers with a square-core are commercially available with traditional round cladding as well with square cladding which provide greater capture area in bundles when put in an array. ${ }^{33}$

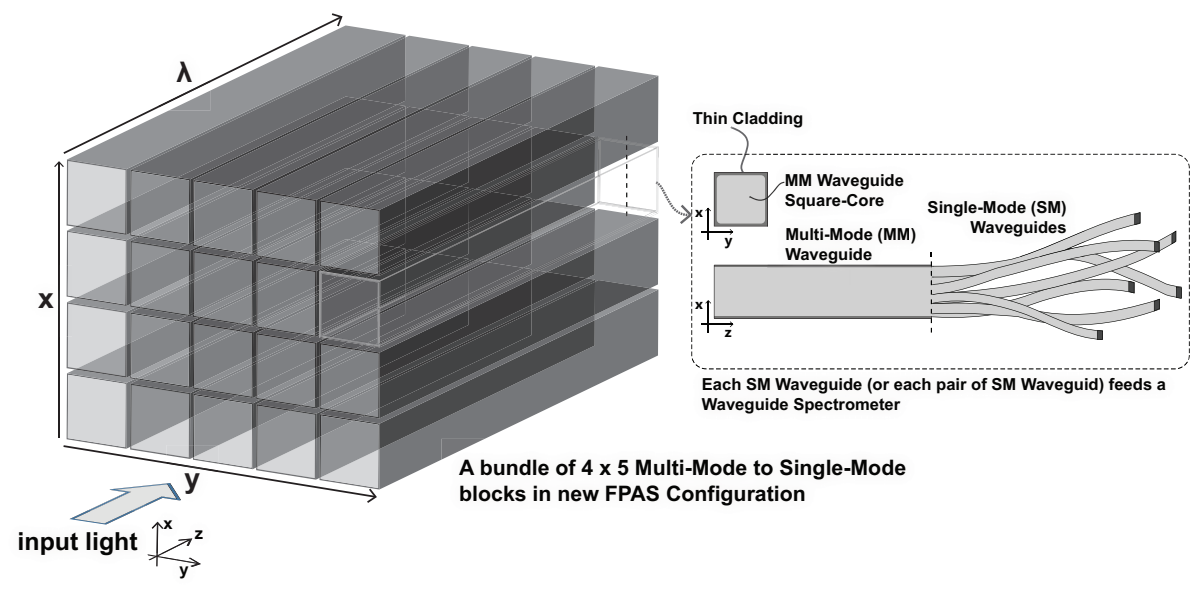

Figure 15. Layout of the innovative FPAS based on the concept of square/rectangular-core multi-mode waveguides feeding waveguide spectrometers. Here the multi-mode square/rectangular-core optical fiber with thin cladding layers occupy the entire focal plane of the telescope where the light from the scene is collected. The rectangular/square-core fiber array accepts almost as much light as a detector array.

In an alternative configuration, a pair of single-mode fibers can feed a single-mode waveguide spectrometer from both sides to form a counter-propagative interference pattern around the center-burst or ZPD inside the waveguide core. Consequently, the ZPD is no longer at the edge of the waveguide but in the middle of the optical path. In this way, the ZPD data is retrievable contrary to the state-of-the-art FPAS design.

As discussed in Sec. 2.2, the access to ZPD data is essential for broadband spectrum applications as the interferogram adjacent to ZPD contains the main broadband information. Thereby, the new FPAS concept introduced in this article has the additional advantage of being adaptable for broadband applications. This alternative configuration is shown in Fig. 16. In an upgraded version, integrated interferogram scanning techniques, e.g. electro-optic actuators, may be embedded in the system to increase the sampling capabilities of 


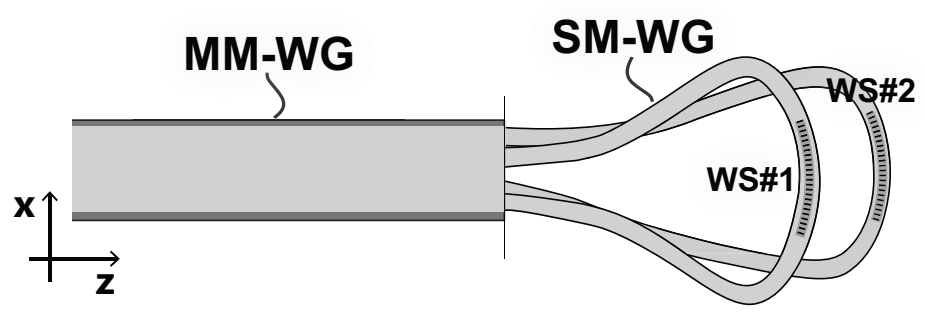

Figure 16. Schematic of a multi-mode waveguide spectrometer with counter-propagative configuration for broadband applications. This configuration can act as a self-standing waveguide spectrometer or as a pixel for the FPAS shown in Fig. 15.

individual waveguide spectrometers yielding a superlative wideband FPAS. ${ }^{34-36}$

\section{CONCLUSION}

In this article, we reported the preliminary results of the FPAS development. We showed that the throughput in the original FPAS design concept is limited due to the limited throughput in the level of individual single-mode waveguides. Hence, the energy coupling efficiency into waveguide spectrometers is less than $50 \%$ in the best case scenario. Throughput is an important factor in waveguide spectrometers especially for imaging applications such as remote sensing, where the collected irradiance at the focal plane of the telescope is extremely faint. We propose to use a bundle of single-mode waveguides, instead of only one, at the focal distance of the optical element. The bundle of single-mode waveguides is fed by a multi-mode waveguide acting as a single pixel of FPAS. A significant advantage of the innovative FPAS configuration based on multi-mode waveguides is that theoretically up to $90 \%$ of the collected photons are coupled into the spectrometer system. In addition to throughput enhancement, since each spatial pixel of FPAS is split into several single-mode fibers, each waveguide spectrometer can be designed to operate in a certain spectral band yielding an enhancement in the system's overall spectral bandwidth. A temporal averaging of sampled interferograms over waveguides would as well increase the SNR in the system. Portable and highly compact sensing FTS devices with low integration speed can be realized when the singlemode waveguide spectrometers are fed by a high-throughput multi-mode waveguide structure. On the other hand, the combination of large arrays of such multi-mode structures in FPAS configuration yields FTS devices with fast integration speed suitable for remote sensing applications. Thereby, the authors believe the application of multi-mode guiding structures to increase the throughput is an effective solution for further developments of compact imaging spectrometers. The appropriate material platform based on which the proposed device can be realized needs to be investigated further.

\section{ACKNOWLEDGMENTS}

This work was funded under European Space Agency Networking/Partnering Initiative (NPI 259-2012) and European Space Agency Technology Research Program (TRP 4000104975/11/NL/NA). The authors thank Philippe Giaccari and Flavio Fontana for their technical support and suggestions during the study.

\section{REFERENCES}

[1] Guldimann, B., Kraft, S.: Focal plane array spectrometer: miniaturization effort for space optical instruments. Proc. SPIE MOEMS and Miniaturized Systems X; 79300O. vol 7930, San Francisco: SPIE MOEMS-MEMS, (2011)

[2] Ferrand, J., Custillon, G., Leblond, G., Thomas, F., Moulin, T., Le Coarer, E., Morand, A., Blaize, S., Gonthiez, T., Benech, P.: Stationary wave integrated fourier transform spectrometer (SWIFTS). Integrated Optics: Devices, Materials, and Technologies XIV vol 7604, p 760414 (2010)

[3] Le Coarer, E., Blaize, S., Benech, P., Stefanon, I., Morand, A., Lérondel, G., Leblond, G., Kern, P., Fedeli, J. M., Royer, P.: Wavelength-scale stationary-wave integrated Fourier-transform spectrometry. Nature Photonics 1 473-478 (2007) 
[4] Fabrice, T., De Mengin, M., Duchemin, C., Le Coarer, E., Bonneville, C., Gonthiez, T., Morand, A., Benech, P., Dherbecourt, J. B., Hardy, E.: High-performance high-speed spectrum analysis of laser sources with SWIFTS technology. Photonic Instrumentation Engineering vol 8992 (International Society for Optics and Photonics) p 89920I (2014)

[5] Bonneville, C., Thomas, F., de Mengin, P., Le Coarer, E., Benech, P., Gonthiez, T., Morand, A., Coutant, O., Morino, E., Puget, R.: SWIFTS: a groundbreaking integrated technology for highperformance spectroscopy and optical sensors. MOEMS and Miniaturized Systems XII vol 8616 (International Society for Optics and Photonics) p 86160M (2013)

[6] Bonneville, C.: High-resolution spectrometers shrink down with SWIFTS. Laser Focus World 50 57-59 (2014)

[7] Cheben, P., Schmid, J. H., Florjańczyk, M., Bock, P. J., Xu, D. X., Janz, S., Delâge, A., Lapointe, J., Lamontagne, B., Post, E., Densmore, A., Albert, J., Hall, T. J., Solheim, B., Scott, A.: Recent Progress in Planar Waveguide Spectrometers. Integrated Photonics and Nanophotonics Research and Applications (Optical Society of America) p IMD4 (2009)

[8] Osowiecki, G. D., Madi, M., Shorubalko, I., Philipoussis, I., Alberti, E., Scharf, T., Herzig, H. P.: Standing wave integrated Fourier transform spectrometer for imaging spectrometry in the near infrared. Imaging Spectrometry XX vol 9611 (International Society for Optics and Photonics) p 96110P (2015)

[9] Zgraggen, E., Scholder, O., Bona, G. L., Fontana, F., Alberti, E., Crespi, A., Osellame, R., Scharf, T., Shorubalko, I.: Optical properties of waveguide-coupled nanowires for sub-wavelength detection in microspectrometer applications. Journal of Optics 17 025801(2015)

[10] Madi, M., Ceyssens, F., Shorubalko, I., Herzig, H. P., Guldimann, B., Giaccari, P.: Lippmann waveguide spectrometer with enhanced throughput and bandwidth for space and commercial applications. Opt. Express 26 2682-2707 (2018)

[11] Guan, T., Ceyssens, F., Puers, R.: An EpoClad/EpoCore-based platform for MOEMS fabrication. J. Micromech. Microeng. 23125005 (2013)

[12] Madi, M.: High spectral resolution waveguide spectrometer with enhanced throughput for space and commercial applications. Lausanne: EPFL, doi:10.5075/epfl-thesis-7087 (2016)

[13] Scholder, O., Jefimovs, K., Shorubalko, I., Hafner, C., Sennhauser, U., Bona, G. L.: Helium focused ion beam fabricated plasmonic antennas with sub-5 nm gaps. Nanotechnology 24395301 (2013)

[14] Akcay, C., Parrein, P., Rolland, J. P.: Estimation of longitudinal resolution in optical coherence imaging. Appl. Opt. 41 5256-5262 (2002)

[15] Hodara, H., Slemon, C.: Throughput and coupling in optical fibers. Appl. Sci. Res. $41203-221$ (1984)

[16] Manzardo, O.: Micro-Sized Fourier Spectrometers. Neuchâtel: University of Neuchâtel (2002)

[17] Goldman, D. S., White, P. L., Anheier, N. C.: Planar waveguide spectrometer. Optoelectronic Devices and Applications vol 1338 (International Society for Optics and Photonics) p 64-69 (1990)

[18] Calvo, M. L., Lakshminarayanan. V.: Optical waveguides: from theory to applied technologies. vol 5, CRC Press (2007)

[19] Florjańczyk, M., Alonso-Ramos, C., Bock, P., Bogdanov, A., Cheben, P., Molina-Fernández, Í., Janz, S., Lamontagne, B., Ortega-Moñux, A., Scott, A.: Development of a Fourier-transform waveguide spectrometer for space applications. Opt. and Quantum Electron. 44 549-556 (2012)

[20] Sinclair, K., Cheben, P., Florjańczyk, M., Quine, B., Scott, A., Solheim, B.: Design of a slab waveguide multiaperture spectrometer for field observations. Can. Aeronaut. Space J. 57 53-58 (2011)

[21] Florjańczyk, M., Cheben, P., Janz, S., Scott, A., Solheim, B., Xu, D. X.: Planar waveguide spatial heterodyne spectrometer. Photonics North 2007 vol 6796 (International Society for Optics and Photonics) p 67963J (2007)

[22] Florjańczyk, M., Cheben, P., Siegfried, J., Scott, A., Solheim, B., Xu, D. X.: Multiaperture planar waveguide spectrometer formed by arrayed Mach-Zehnder interferometers. Opt. Express 1518176 18189 (2007)

[23] Delâge, A., Cheben, P., Florjanczyk, M., Janz, S., Lamontagne, B., Lapointe, J., Scott, A., Solheim, B., Xu, D. X.: Static Fourier-transform waveguide spectrometers. Transparent Optical Networks. ICTON'09. 11th International Conference (IEEE) p 1-4 (2009) 
[24] Bland-Hawthorn, J., Ellis, S., Leon-Saval, S., Haynes, R., Roth, M., Löhmannsröben, H., Horton, A., Cuby, J., Birks Tim, A., Lawrence, J.: A complex multi-notch astronomical filter to suppress the bright infrared sky. Nature Commun. 2 581(2011)

[25] Leon-Saval, S. G.: Multimode photonics: Harnessing optical transition devices. European Conference on Optical Communication (ECOC) (IEEE) p 1-3 (2015)

[26] Birks Tim, A., Mangan, B., Díez, A., Cruz, J., Murphy, D.: Photonic lantern spectral filters in multicore fibre. Opt. Express 20 13996-14008 (2012)

[27] Leon-Saval, S. G., Argyros, A., Bland-Hawthorn, J.: Photonic lantern mode evolution: A multicore geometry study. Communications and Photonics Conference and Exhibition (ACP) (IEEE) p 697-698 (2010)

[28] Schiappelli, F., Kumar, R., Prasciolu, M., Cojoc, D., Cabrini, S., De Vittorio, M., Di Fabrizio, E.: Efficient fiber-to-waveguide coupling by a lens on the end of the optical fiber fabricated by focused ion beam milling. Microelectronic Engineering 73 397-404 (2004)

[29] Tiecke, T., Nayak, K., Thompson, J., Peyronel, T., De Leon, N., Lukin, M.: Efficient fiber-optical interface for nanophotonic devices. Optica 2 70-75 (2015)

[30] Barry, T., Rode, D., Krchnavek, R.: Highly efficient coupling between single-mode fiber and polymer optical waveguides. IEEE Trans. Compon. Packag. Manuf. Technol. 20 225-228 (1997)

[31] Alferness, R., Ramaswamy, V., Korotky, S., Divino, M., Buhl, L.: Efficient single-mode fiber to titanium diffused lithium niobate waveguide coupling for $\lambda=1.32 \mu \mathrm{m}$. IEEE J. Quantum Electron. 18 1807-1813 (1982)

[32] Phoenix Photonics: D-shaped side-polished optical fibers. Phoenix Photonics website: http://www.phoenixphotonics.com (2017)

[33] Schuberts, F., Hoben, A., Bakhshpour, K., Provost, C.: Square fibers solve multiple application challenges. Photonics Spectra 45 38-41 (2011)

[34] Pohl, D., Reig Escalé, M., Madi, M., Brotzer, P., Kaufmann, F., Sergeyev, A., Meier, U., Alberti, E., Giaccari, P., Grange, R.: Integrated Waveguide Fourier Transform Spectrometer on Thin-Film Lithium Niobate. CLEO/Europe: Conference on Lasers and Electro-Optics, 23-27 June 2019, Munich, Germany (2019)

[35] Reig Escalé, M, Pohl, D., Madi, M., Brotzer, P., Kaufmann, F., Sergeyev, A., Meier, U., Alberti, E., Grange, R.: Integrated Electro-Optic Spectrometers on Thin-Film Lithium Niobate. CLEO/US: Conference on Lasers and Electro-Optics, 5-10 May 2019, San Jose, California, USA (2019)

[36] Pohl, D., Reig Escalé, M., Madi, M., Kaufmann, F., Brotzer, P., Sergeyev, A., Guldimann, B., Meier, U., Alberti, E., Grange, R.: An integrated broadband spectrometer on thin-film lithium niobate. Nat. Photonics 14, 24-29 (2020) 